\title{
Effectiveness of a Cognitive-Behavioral Intervention in Burnout: Case Series
}

\section{Lechuga Gómez LR and Álvarez Garcia HB*}

${ }^{1}$ UMAE Clinical Psychologist, General Hospital Dr. Gaudencio Gonzalez Garza, Mexico ${ }^{2}$ Postgraduate program in Medical, Dental and Health Sciences, National Autonomous University of Mexico, Mexico

*Corresponding author: Horacio Balam Álvarez García, Postgraduate program in Medical, Dental and Health Sciences, National Autonomous University of Mexico, Buenavista 13, Col. Buenavista, Alcaldía Cuauhtémoc, CDMX, Mexico, Email: hbgarcia_mosh11@hotmail.com

\section{Case Report \\ Volume 5 Issue 2}

Received Date: August 28, 2021

Published Date: October 05, 2021

DOI: $10.23880 / \mathrm{mhrij}-16000152$

\section{Abstract}

Background: Burnout syndrome is a recurring problem in workers from different areas that causes cognitive, emotional, physical and behavioral disorders that diminish the quality of life of those who suffer from it. It has been reported that there is a risk of developing anxiety and depression if proper treatment is not provided. Objective: to evaluate the effect of a cognitive behavioral intervention in the burnout syndrome, comorbidities such as: anxiety, depression and improving the quality of life in two medical workers from Mexico City.

Method: A quantitative investigation was carried out in a case report mode with an intrasubject design with pretreatment and post treatment measurements.

Results: In the statistical analysis, no changes were observed in the scores that were statistically significant ( $\mathrm{p} \geq 0.05)$, however, in the visual inspection changes in the scores of the BDI (Pre $=14$, Post $=4)$, BAI (Pre $=27$, Post $=1)$, MBI $($ Pre $=88$, Post $=52)$ and Q-SLE-Q (Pre $=21$, Post $=31)$ for case 1 , although in case 2 no changes were observed in two of the instrument: BDI (Pre $=11$, Post $=12)$ Q-LES-Q $($ Pre $=32$, Post $=33)$ and a partial decrease in the other two BAI $($ Pre $=18$, Post $=9)$ and MBI $($ Pre = 60 , Post $=53$ ).

Conclusion: From the observed changes, it is inferred that the intervention was possibly effective for one of the cases. However, it is suggested to improve the research design to obtain better results.

Keywords: Burnout; Anxiety; Depression; Quality of Life; Cognitive Behavioral Intervention

Abbreviations: IMSS: Mexican Institute of Social Security; BAI: Beck Anxiety Inventory; MBI: Maslach Burnout Inventory; CBI-B: Cognitive Behavioral Intervention for Burnout.

\section{Introduction}

Burnout is a response to chronic emotional stress with three components; emotional and/or physical exhaustion, low job performance and excess depersonalization [1].
Burnout is considered an occupational disease that causes deterioration of the physical and mental health of individuals, which must be controlled since in the long term it can affect not only the lives of health professionals but also the health system in general [2]. Among the job characteristics associated with burnout, high volume of work, conflict and role ambiguity, low predictability, lack of participation and social support, and experience of injustice stand out [3]. Within the clinical manifestations associated with burnout, 3 large groups are considered: 


\section{Mental Health \& Human Resilience International Journal}

1. Psychiatric (depression and anxiety),

2. Somatic and

3. Organizational (deterioration of labor relations and quality of care [4].

Currently, cognitive behavioral therapy has been an empirically validated procedure in this disorder [5]. Among the components used, relaxation techniques, training in social skills, positive self-verbalization, cognitive restructuring, intervention in rumination, training in time management and relapse prevention stand out [6]. Derived from the COVID-19 pandemic, an increase in burnout has been observed in firstlevel health personnel under this scenario [7], the present investigation sought to reduce Burnout in medical personnel in a hospital for COVID patients in Mexico City.

\section{Method}

A quantitative investigation was carried out in a case report mode with an intrasubject design with pretreatment and post treatment measurements [8].

\section{Presentation of Patients}

Due to the health measures derived from the COVID-19 pandemic, an invitation was made to the medical staff of the Mexican Institute of Social Security (IMSS). UMAE General Hospital Dr. Gaudencio González Garza "La Raza" National Medical Center who is working and presented some type of emotional discomfort that was affecting his work performance to go to the psychology department, finally only two workers decided to participate.

\section{Case 1}

LL. He is a 48-year-old man, married with 2 children, a medical specialist by profession. He approached the psychology department derived from physical and psychological discomfort due to working hours in the hospital in the care of patients with COVID-19 with an average of 10 hours a day.

\section{Case 2}

LS. She is a 36-year-old woman, single, lives in her own home, and is a specialist doctor by profession. He approached the psychology department derived from physical and psychological discomfort due to working hours in the hospital caring for patients with COVID-19 with an average of 18 hours a day.

\section{Instruments}

The following instruments were used
- Beck Depression Inventory (BDI-II). Self-report that provides a measure of depression symptoms in people ages 13 to 65 . Inventory is made up of two factors: (a) cognitive-affective dimension of 12 items and (b) somatic-motivational dimension. The item format is Likert type from 0 to 3 . The rating is obtained through the sum of the responses to the 21 items with a range from 0 to 63 points. It is interpreted in intervals of: $0-13$, minimal depression; 14-19, mild depression; 20-28, moderate depression; and 29-63, severe depression. The results showed that the internal consistency in the total Mexican sample was satisfactory, both for the general factor $(\propto=0.90)$ [9].

- $\quad$ Beck Anxiety Inventory (BAI). self-applied instrument made up of 21 reagents. It is scored on a Likert-type scale from 0 to 3 . The total score is obtained from the sum of all the items and ranges from 0 to 63. The norms for interpretation range: from 0 to 5 points, minimal anxiety; 6 to 15, mild anxiety; from 16 to 30 points, moderate anxiety, and from 31 to 63 , severe anxiety. The Mexican version for adults is characterized by high internal consistency $(\alpha=0.83)$ [10].

- Maslach Burnout Inventory (MBI). 22-item selfadministered questionnaire. It is scored on a Likerttype scale from 1 (never) to 7 (daily). It is made up of three factors: the Subscale for Emotional Exhaustion, the Subscale for Depersonalization and Low Personal Achievement at Work (PA). It is interpreted according to the scores for each factor; in the first two the high scores correspond to increased feelings of burning, while in the third the low scores correspond to high feelings of burning. One of the validations in Mexico obtained a Cronbach's alpha of .65 for the entire scale and a $41.6 \%$ explained variance [11].

- Questionnaire on Quality of Life: Satisfaction and Pleasure (Q-LES-Q) Self-applied instrument consisting of 93 items, scored from never to very often. It explores the satisfaction and pleasure experienced in the following 8 areas: Physical state of health (state of mind, Work, home activities, class/course assignments, free time activities, social relationships, general activities, and satisfaction with the overall medication, finally the instrument presents a Cronbach's alpha of 89 [12].

\section{Intervention}

Once the informed consents were signed, the cognitive behavioral intervention for burnout (CBI-B) consisted of a total of 10 sessions with an average duration of 90 minutes each, given once a week, divided into 2 evaluation sessions and 8 treatment. The techniques used were: 


\section{Psychoeducation}

They were given information about what Burnout is, the physical, emotional and cognitive indicators to finally explain the logic of the treatment.

\section{Relaxation Techniques (Muscle Relaxation, Diaphragmatic Breathing, Autogenic Training)}

Through the exercises it was sought to reduce the psychophysiological activation derived from the Burnout.

\section{Cognitive Restructuring}

Patients were taught to identify their dysfunctional thoughts associated with their Burnout persona. Through searching for evidence and Socratic debate, more adaptive thinking was taught.

\section{Training in Social Skills}

Through exercises in session, they were taught to communicate effectively and assertively their thoughts and emotions, with the aim of reducing conflicts with their peers.

\section{Time Management}

Using an agenda, patients were taught to resume leisure activities that would help them relax.

\section{Analysis of Data}

The non-parametric Wilcoxon tests were used for the significance of the changes in the inventory scores with the support of the SPSS version 22 software. In addition, a visual analysis of the changes in the graphs obtained was performed.

\section{Results}

Within the analysis of the Wilcoxon test, no statistically significant change was obtained in any of the inventories of both participants (Table 1).

\begin{tabular}{|c|c|c|}
\hline Instrument & z-value & Sig. Asymptotic (bilateral) \\
\hline BDI & -0.447 & 0.655 \\
\hline BAI & -1.342 & \multirow{2}{*}{0.18} \\
\hline MBI & -1.342 & \\
\hline QLESQ & $-1.342^{\mathrm{c}}$ & \\
\hline
\end{tabular}

Note: DI: Beck Depression Inventory. BAI: Beck Anxiety Inventory. MBI: Maslach Burnout Inventory. Q-LES-Q: Quality of Life Questionnaire: Satisfaction and Pleasure.

Table 1: Statistic analysis.

On the other hand, the visual inspection allows us to observe that in Case 1 there were changes in all the instruments. Observing a decrease in BDI (Pre $=14$, Post $=$ $4)$, BAI (Pre $=27$, Post $=1)$, MBI (Pre $=88$, Post $=52$ ) that according to the dimensions represents an improvement in clinical problems. Finally, an increase is observed in the Q-SLE-Q scores (Pre $=21$, Post $=31$ ), interpreted as an improvement in the quality of life of the person (Figure 1).

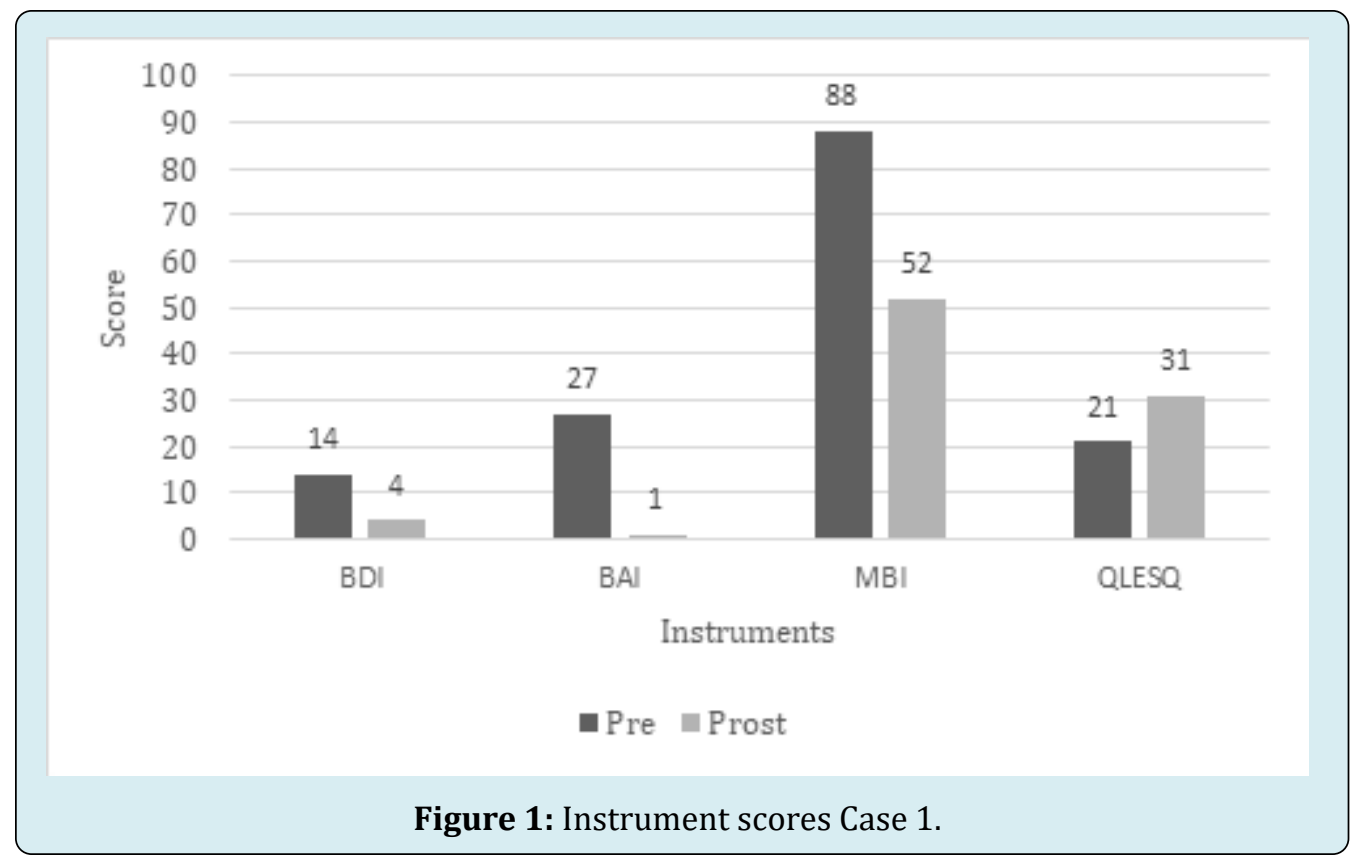




\section{Mental Health \& Human Resilience International Journal}

In contrast, in the visual inspection of Case 2, contrasting changes are observed in all instruments, with an increase of only one point in BDI (Pre $=11$, Post $=12$ ) and Q-LES-Q (Pre $=32$, Post $=33$ ) that would not have clinical relevance and $\mathrm{a}$ decrease in BAI (Pre $=18$, Post $=9)$ and MBI (Pre $=60$, Post $=$ $53)$, which according to the instruments would show clinical improvement (Figure 2).

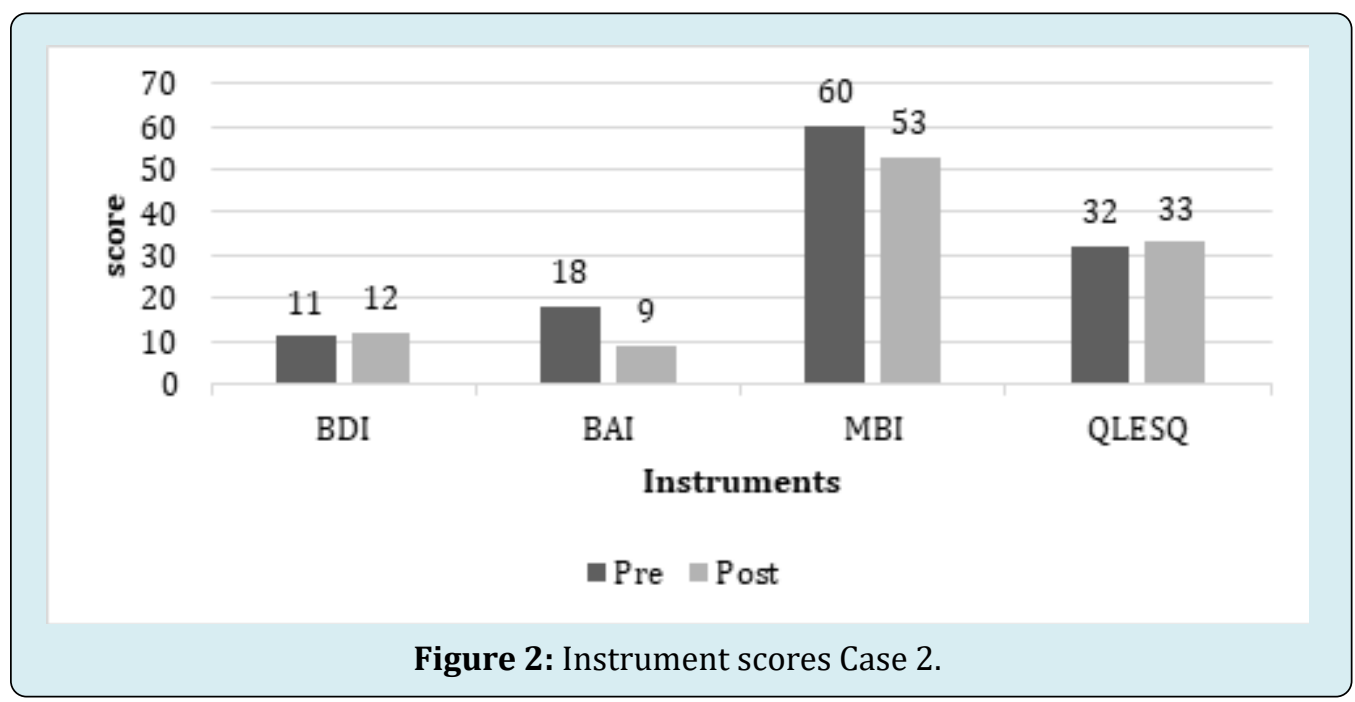

\section{Discussion}

The various studies that have been carried out reveal the complexity of burnout; hence the importance of generating comprehensive programs for prevention and intervention. The cognitive-behavioral model allows the development of a brief, cost-effective treatment focused on the presentmoment, in order to improve individual conditions and expand the repertoire of social skills applied in the workplace [5]. The intervention was developed during the COVID-19 pandemic in the period from December 2020 to early February 2021, in which there was an increase in infections that led to saturation in hospital occupation, enabling various areas to be able to provide care to a greater number of patients simultaneously. In contrast, there was a decrease in medical personnel due to multiple infections among health sector workers. It has been reported that this situation has led to an increase in disorders such as anxiety, depression and stress $[13,14]$, which would explain the minimal changes in the scores observed in case 2 . However, timely intervention in pandemic contexts will aim to improve the mental health of personnel in COVID areas [15,16], which is reflected in the improvements observed in Case 1. A relevant aspect to consider is the differences sociodemographic in each case, since the protective factors to face the work context varied considerably, which influenced the effectors of CHF-B.

\section{Conclusion}

Under this analysis it is suggested to improve the individual methodological designs by designs $\mathrm{n}=1$ for future individual interventions or to homogenize the sample to carry out group interventions.

\section{Interest Conflict}

The authors are reported a conflict of interest.

\section{References}

1. Morales LS, Hidalgo Murillo LF (2015) Burnout syndrome. Legal Medicine of Costa Rica 32(1): 119-124.

2. Gil P (1999) Theoretical perspectives and interpretive models for the study of burnout syndrome. Annals of Psychology 15(2): 261-268.

3. Carlin M, Garces E (2010) Burnout syndrome: "Historical evolution from the work context to the sports field". Annals of Psychology 26(1): 169-180.

4. Esper RC, Hernandez KG, Espinoza I (2012) Burnout syndrome in medical practice. Med Int Mex 28(6): 579584.

5. West CP, Dyrbye LN, Erwin PJ, Shanafelt TD (2016) Interventions to prevent and reduce physician burnout: a systematic review and meta-analysis. Lancet 388(10057): 2272-2281.

6. Ruwaard J, Lange A, Schrieken B, Dolan CV, Emmelkamp $P(2012)$ the Effectiveness of Online Cognitive Behavioral Treatment in Routine Clinical Practice. PLOS ONE 7(7): e40089.

7. Barello S, Palamenghi L, Graffigna G (2020) Burnout 


\section{Mental Health \& Human Resilience International Journal}

and somatic symptoms among frontline healthcare professionals at the peak of the Italian COVID-19 pandemic. Psychiatry research 290: 113129.

8. Garcia MA, Seco GV (2015) Research designs in psychology. Madrid: Pyramid Editions.

9. Aranda BE, Alvarez CD, Hernandez RL, Ramirez MG (2014) Psychometric properties of the bifactorial model of the BDI-II (Spanish version) in Mexican samples of the general population and university students. Universities Psychology 14(1): 125-136.

10. Guillén DB, González CR (2019) Psychometric properties of the Beck Anxiety Inventory in Mexican asthmatic adults. Psychology and Health 29(1): 5-16.

11. Aranda BC, Pando MM, Salazar EJG (2016) Reliability and validation of the Maslach Burnout Inventory (Hss) scale in workers from western Mexico. Uninorte Health Magazine 32(2): 218-227.

12. Hauser MP, Garcia HD, Leporati J (2020) Reliability and validity analysis of the Quality of Life, Satisfaction and Pleasure Questionnaire (Q-LES-Q) in Public Health workers. Revisit Inter psychology/Inter J Psychology 54(3): 1154.

13. Santamaria MD, Etxebarria NO, Rodriguez IR, Alboniga Mayor J, Gorrotxategi MP (2021) Psychological impact of COVID-19 in a sample of health professionals SpanishPsychological impact of COVID-19 on a sample of Spanish health professionals. J Psychia Ment Health 14(2): 106-112.

14. Garcia Iglesias JJ, Salgado JG, Pereira JM, Rivera JF, Murillo DA, et al. (2020) Impact of SARS-CoV-2 (Covid-19) on the mental health of healthcare professionals: a systematic review. Rev Esp Salud Publica 94: e202007088.

15. Saeki S, Shimato M (2021) Mental Health Support for the Current and Future Medical Professionals during Pandemics. JMA 4(3): 281-283.

16. Cairns P, Aitken G, Pope LM, Cecil JE, Cunningham KB, et al. (2021) Interventions for the well-being of healthcare workers during a pandemic or other crisis: scoping review. BMJ open 11(8): e047498. 